\title{
EMI: EFFICIENT TEMPORAL PHASE ESTIMATION AND ITS IMPACT ON HIGH-PRECISION INSAR TIME SERIES ANALYSIS
}

\author{
Homa Ansari ${ }^{1}$, Francesco De Zan ${ }^{1}$, Giorgio Gomba ${ }^{1}$, Richard Bamler ${ }^{1,2}$ \\ ${ }^{1}$ Remote Sensing Technology Institute (IMF), German Aerospace Center (DLR) \\ ${ }^{2}$ Chair of Remote Sensing Technology (LMF), Technical University of Munich (TUM)
}

\begin{abstract}
Multitemporal phase estimation aims at the exploitation temporal data redundancy within the SAR time-series to reduce the impact of inherent stochastic and systematic interferometric phase inconsistencies [1] for distributed scatterers (DS). The consistent phase-series estimated as such is further utilized to retrieve the underlying geophysical and atmospheric signals. Therefore, the precision and interpretability of the retrieved physical signals from the DS is governed by the performance of the phase estimators. Different approaches to phase estimation calls for the investigation of their performance. Here we explain the discrepancy among the different approaches in terms of their underlying covariance model and introduce our recently proposed estimator named EMI [2]. Bridging between different approaches via revised mathematical formulation of phase estimation, EMI enhances the estimation precision and computational efficiency of the temporal phase estimation. The performance of different phase estimators is brought into attention via simulation analysis. Using Sentinel-1 time series over the North and East Anatolian Faults, wide area performance analysis is further carried out and will be presented.
\end{abstract}

Index Terms - maximum likelihood estimation, estimation efficiency, sample covariance matrix, interferometric synthetic aperture radar, low-rank approximation

\section{INTRODUCTION}

Interferometric Synthetic Aperture Radar (InSAR) is an established geodetic technique for retrieval of Earth surface deformation. The precision and accuracy of InSAR in geodetic signal retrieval depend on the effective reduction of the error sources from the interferograms. Among such sources is the signal decorrelation in multipass SAR acquisitions. It occurs due to abrupt changes or stochastic variation of the position and/or dielectric properties of the sub resolution scatterers between acquisitions, introducing stochastic noise and systematic error among the interferograms of a SAR time series. Referred to as stochastic and systematic phase inconsistencies [1], such effects compromise the precision and interpretability of the retrieved geodetic signals. In circumvent- ing these effects, geodetic signal retrieval may be limited to phase-stable persistent scatterers (PS). In areas where PSs are scarce, the exploitation of natural distributed scatterers (DS) is inevitable. Temporal phase estimators are introduced to improve the Signal to Noise Ratio (SNR) of the DSs within the SAR time series. In contrast to the use of such estimators, the temporal exploitation of SAR data may be limited to partially coherent small baseline interferograms. The latter approach simplifies geodetic signal retrieval for DSs, at the expense of reduced precision.

Temporal phase estimators allow the retrieval of a time series of consistent interferograms from all possible inconsistent interferograms within a SAR time series. In doing so, they effectively reduce the stochastic phase noise. Exploiting the temporal data redundancy, they are theoretically expected to be less susceptible to systematic inconsistencies as well [1].

Different approaches to temporal phase estimation may be categorized to two groups, namely the Maximum Likelihood Estimators (MLE) and the approximate Eigendecompositionbased (EVD) estimators. Here we shed light on the underlying model and pros and cons of these approaches while introducing a new MLE which overcomes the disadvantages of the aforementioned approaches [2]. Called Eigendecomposition-based Maximum likelihood estimator of Interferometric phase (EMI), the proposed estimator is shown to be efficient both in terms of estimation precision and computational cost.

\section{TEMPORAL PHASE ESTIMATION}

Temporal phase estimation is considered for a DS region. Allow $\Omega$ as an ensemble of statistically similar pixels pertaining to this region, a spatiotemporal aggregation of its corresponding complex valued pixels reads as: $Z \in \mathbb{C}^{n \times l}$, where $l$ refers to the spatial and $n$ to the temporal domain. Based on the central limit theorem, $Z$ follows the zero-mean $n$ variate complex circular Gaussian distribution. Under the assumption of validity of this distribution, the second order moment of $Z$ suffices for the full description of data statistics. The sample coherence matrix (SCM), hereafter denoted by $C \in \mathbb{C}^{n \times n}$, is the MLE of the second order moment. 
The SCM presents the interferometric content of the DS region concisely and sufficiently. It is a Hermitian matrix whose off diagonal elements pertain to the $n(n-1) / 2$ interferograms within the SAR time series.

Recalling that temporal phase estimation retrieves the consistent common-master interferograms from the time series, it may be reformulated into the problem of modeling the SCM [3, 2].

\subsection{SCM information content}

Allow the decomposition of the complex SCM to:

$$
\begin{aligned}
& C=\Gamma \circ \mathrm{I}_{\Omega}, \\
& \Gamma_{i k}=\left|C_{i k}\right|, \\
& \left(\mathrm{I}_{\Omega}\right)_{i k}=\exp \left(j \Delta \phi_{i k}\right),
\end{aligned}
$$

where $\circ$ represents the element-wise Hadamard product, $j$ is the imaginary unit and the subscripts refer to the elements of the associated matrix. For a generic DS the coherence matrix $\Gamma$ is a full-rank symmetric matrix, its elements represent the coherence of the associated interferogram. $\mathrm{I}_{\Omega}$ on the other hand is a low-rank matrix. In the absence of stochastic and systematic phase inconsistencies, it degenerates to a rank-1 dyad. The sufficiency of a rank- 1 dyad for description of $I_{\Omega}$ indicates that the multi-master interferograms are redundant and the geodetic signal is contained in a series of commonmaster interferograms. The sought for this rank-1 dyad is the purpose of phase estimators.

\subsection{Different Approaches to SCM Modeling}

\subsubsection{Phase Triangulation Algorithm (PTA)}

PTA is the pioneering approach in temporal phase estimation $[4,5]$. Its proposed SCM model reads as:

$$
\begin{aligned}
\Sigma & =\Gamma \circ \boldsymbol{\psi} \boldsymbol{\psi}^{H}, \\
\text { with } \quad \boldsymbol{\psi} & =\exp (j \boldsymbol{\phi}) .
\end{aligned}
$$

Here and after $\phi \in \mathbb{R}^{n \times 1}$ represents the sought consistent interferogram series, bold symbols indicate vectors. According to this model, the coherence matrix remains intact while the $I_{\Omega}$ is approximated by a rank- 1 dyad. Fitting this model through the maximization of the Wishart distribution [3], PTA may be shown to be a MLE $[4,5]$.

Being MLE, PTA is asymptotically an optimum estimator; it is unbiased and its variance attains the Cramer-Rao Lower Bound (CRLB) for temporal phase estimation [6]. The PTA is however sensitive to the estimation performance of the coherence matrix $\Gamma$ [7]. The latter is notoriously known to be sub-optimum for low coherence level and small $l$. PTA estimates the phases through a non-linear optimization scheme with subjective choice of initialization [2]. Therefore its computational expense poses a challenge to Big Data processing [7].

\subsubsection{Eigendecomposition based approaches (EVD)}

Retrieving the consistent interferograms by means of Eigendecomposition of the complex covariance or complex coherence matrix was proposed by [8] and [9], respectively. In the context of temporal phase estimation, we formulate the underlying SCM model of EVD approaches as:

$$
\begin{aligned}
\Sigma & =\lambda \boldsymbol{\alpha} \boldsymbol{\alpha}^{T} \circ \boldsymbol{\psi} \boldsymbol{\psi}^{H}, \\
\text { with } \quad & \boldsymbol{\alpha} \in \mathbb{R}^{n \times 1} .
\end{aligned}
$$

According to this model, not only the low-rank $\mathrm{I}_{\Omega}$ but also the full-rank coherence matrix $\Gamma$ are approximated by rank-1 dyads. The second approximation is mathematically unjustifiable.

Using Eigendecomposition, EVD approaches may enjoy an arsenal of highly efficient numerical recipes for the task of phase estimation. Therefore, they are computationally efficient and well suited to the processing of long SAR time series. However, the EVD's model inadequacy affects the estimation efficiency of these estimators as compared to MLEs and the CRLB [2]. EVDs therefore provide an approximate solution to temporal phase estimation.

\subsubsection{The proposed approach: EMI}

Comparing the pros and cons of the PTA and EMI, a trade-off is observed between the estimation and computational efficiency. To optimize this trade-off and overcome the limitations of the latter approaches, we increase the model complexity for estimation of SCM by proposing:

$$
\Sigma=\Gamma \circ \boldsymbol{\psi} \boldsymbol{\psi}^{H} \circ \lambda \boldsymbol{\alpha} \boldsymbol{\alpha}^{T}
$$

This model resembles the PTA model with a distinct difference. It allows a second rank-1 dyad of $\lambda \boldsymbol{\alpha} \boldsymbol{\alpha}^{T}$, which is seemingly redundant in comparison to Eq. (1). The latter rank-1 dyad serves a purpose when $\Gamma$ matrix is erroneous. It allows for the calibration of $\Gamma$ or in other words relaxes the strict model proposed by PTA. Furthermore, in order to gain computational efficiency, the MLE based on this model is reformulated into a Lagrangian [2] and solved via the Eigendecomposition of the matrix product: $\Gamma^{-1} \circ C$.

The proposed approach is well suited to high-precision estimation of the consistent interferograms. It outperforms the state-of-the-art. However, the increased model complexity may be insufficient, should the estimated $\Gamma$ have severely degraded performance compared to its nominal behavior [2]. The latter disadvantage holds for the EVD and PTA approaches as well.

\section{THE IMPACT OF EMI: SIMULATION ANALYSIS}

The estimation efficiency of temporal phase estimators is governed by the coherence of the exploited interferograms [6]. 

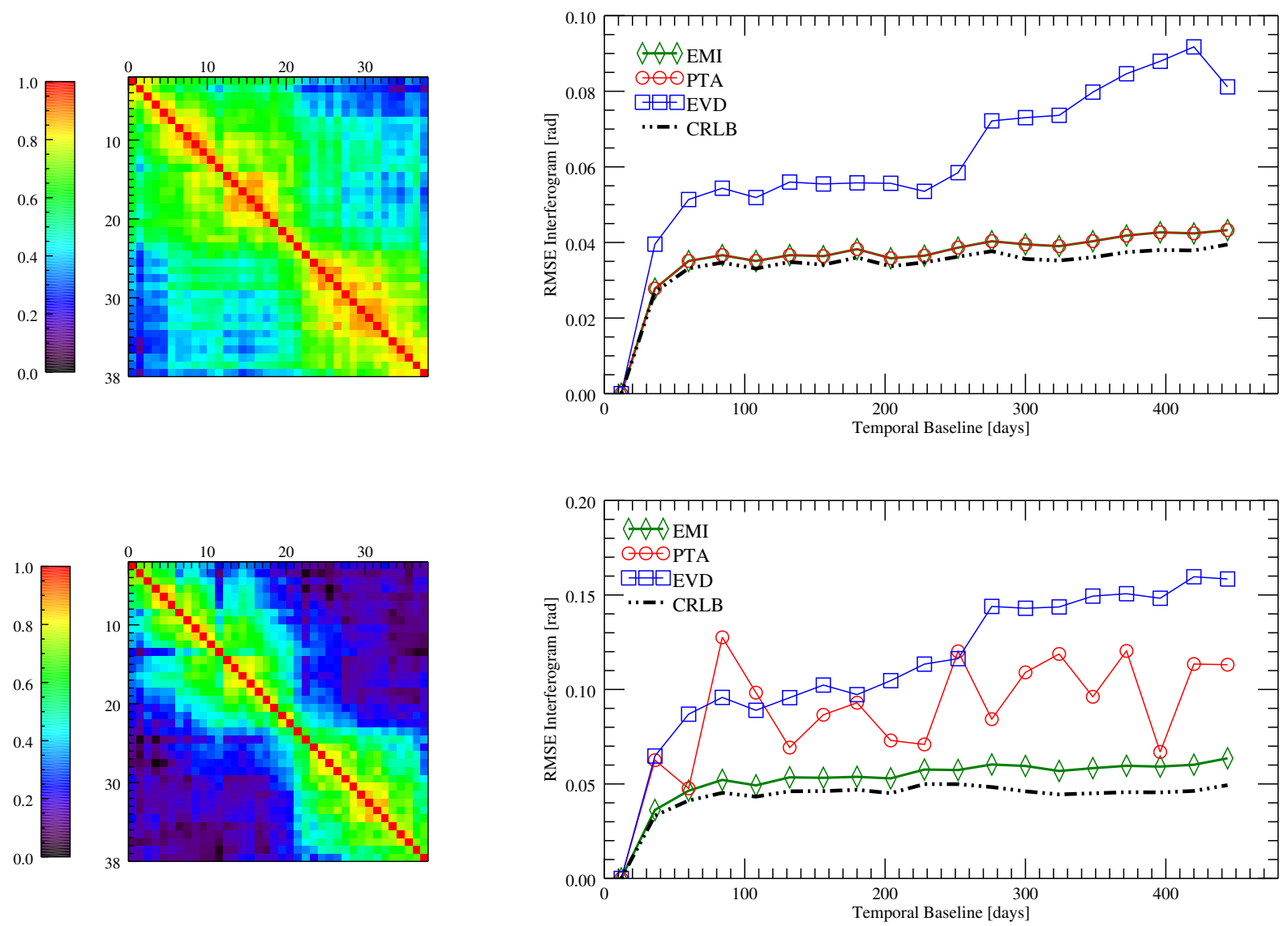

Fig. 1: Performance assessment of different estimators compared to CRLB using simulated cases: (left) the considered coherence matrices for simulations, (right) the RMSE of estimated consistent interferograms for different approaches. Dependent on the estimation efficiency of the coherence matrix, the two MLE approaches of EMI and PTA perform identically close to the CRLB. Is the coherence estimation suboptimum (as in the bottom row), EMI gains in phase estimation efficiency thanks to its increased model complexity. Incorporating an approximate SCM model, the EVD approach is observed to be sub-optimum as its performance deviates from the CRLB.

For the demonstration of this dependency as well as the behavior of the reviewed estimators, we choose two coherence structures here. These coherence matrices are selected from real data processing of Sentinel-1 data. Based on the chosen coherence matrices and assuming the CCG distribution, time series of 38 SAR acquisitions each containing an ensemble of $l$ statistically similar samples is simulated. A linear deformation signal with velocity of $1 \mathrm{~mm} /$ year is assumed for the simulations. The simulated complex ensemble is used in the evaluation of SCM and further in temporal phase estimation with PTA, EVD and EMI approaches. The Root Mean Square Error (RMSE) of the estimated phases compared to the simulated deformation phase is taken as a measure for the performance of phase estimators. This measure encapsulates both the bias and the variance of the estimators. RMSE is evaluated based on 3000 trials of the experiment for each estimator. The CRLB is provided as a lower bound for estimation error.
Fig. 2 depicts the performance analysis for the two cases. In the first case (top row), as seen from the coherence matrix, the majority of the interferograms are moderately coherent, rendering the precise estimation of coherence matrix. In the event of precise estimation of the coherence matrix, the EMI's considered calibration dyadic is redundant and the two MLEbased estimators are expected to perform identical. This expectation is corroborated by the RMSE plot, as the PTA and EMI are shown to identically attain to the lowest error bound provided by CRLB. The performance of the EVD estimator is however seen to be compromised due to its model insufficiency.

In the second simulation case (Fig. 2, bottom row), we deliberately chose a coherence matrix which partially decays to zero level. For such coherence level, the estimation of the coherence matrix is known to be suboptimum [10]. Comparing the performance of the three estimators in this case reveals 
the sensitivity of the PTA approach to the suboptimum estimation of coherence matrix. The EMI however is capable of retaining a performance close to the CRLB, thanks to the consideration of the calibration dyad in its SCM model. Being an approximate approach, EVD is expected and shown to deviate from the CRLB.

\section{EXPERIMENTS WITH REAL DATA}

The performance of the different phase estimators in processing 2-year time series of Sentinel-1 data has been previously carried out on the volcanic island of Vulcano [2]. Result shows the decrease of phase estimation variance specially for short temporal baseline interferograms while circumventing PTA's convergence problems in the optimization [2].

In this work the intention is to demonstrate the performance analysis in wide area processing using Sentinel-1 data; as we previously carried out in [11]. The focus is on evaluation of estimation bias and variance. The initial analysis confirms the conclusions drawn from the Vulcano test site. Further analysis is ongoing and will be presented at the conference.

\section{SUMMARY AND CONCLUSION}

The discrepancies of different temporal phase estimators for interferogram stacks have been formulated in terms of their equivalent SCM model. EMI approach is proposed to allow for improvement of this model via the addition of a calibration dyadic. The latter relaxes the strict model of PTA in order to arrive at more efficient phase retrieval at low computational cost.

Investigating the impact of different phase estimators in processing large areas with variety of land covers allows to gain more insight into the potential and limitation of the phase estimators. An example of such analysis was previously reported over Trans-Mexican Volcanic Belt [11]. Further wide area processing results will be presented at the conference.

The impact of bias and efficiency of phase estimation on deformation signal is a closely related topic to multitemporal phase estimation. We embarked on the quantitative evaluation of phase bias and variance in [11]. Although crucial in the reliability assessment of deformation estimation, such analysis are mostly overlooked by the InSAR community. The latter study is extended here by investigating the inevitable impact of phase estimation quality on deformation maps. The result of such investigations will be presented at the conference.

\section{REFERENCES}

[1] Francesco De Zan, Mariantonietta Zonno, and Paco Lopez-Dekker, "Phase Inconsistencies and Multiple Scattering in SAR Interferometry," IEEE Transactions on Geoscience and Remote Sensing, vol. 53, no. 12, pp. 6608-6616, Dec. 2015.

[2] Homa Ansari, Francesco De Zan, and Richard Bamler, "Efficient Phase Estimation for Interferogram Stacks," IEEE Transactions on Geoscience and Remote Sensing, vol. 56, no. 7, pp. 4109-4125, May 2018.

[3] Ning Cao, Hyongki Lee, and Hahn Chul Jung, "Mathematical Framework for Phase-Triangulation Algorithms in Distributed-Scatterer Interferometry," IEEE Geoscience and Remote Sensing Letters, vol. 12, no. 9, pp. 1838-1842, Sept. 2015.

[4] Andrea Monti Guarnieri and Stefano Tebaldini, "On the Exploitation of Target Statistics for SAR Interferometry Applications," IEEE Transactions on Geoscience and Remote Sensing, vol. 46, no. 11, pp. 3436-3443, Nov. 2008.

[5] Alessandro Ferretti, Alfio Fumagalli, Fabrizio Novali, Claudio Prati, Fabio Rocca, and Alessio Rucci, "A New Algorithm for Processing Interferometric Data-Stacks: SqueeSAR," IEEE Transactions on Geoscience and Remote Sensing, vol. 49, no. 9, pp. 3460-3470, Sept. 2011.

[6] A.M. Guarnieri and S. Tebaldini, "Hybrid Cramr-Rao Bounds for Crustal Displacement Field Estimators in SAR Interferometry," IEEE Signal Processing Letters, vol. 14, no. 12, pp. 1012-1015, Dec. 2007.

[7] Homa Ansari, Francesco De Zan, and Richard Bamler, "Sequential Estimator: Toward Efficient InSAR Time Series Analysis," IEEE Transactions on Geoscience and Remote Sensing, vol. 55, no. 10, pp. 5637-5652, Oct. 2017.

[8] Gianfranco Fornaro, Simona Verde, Diego Reale, and Antonio Pauciullo, "CAESAR: An Approach Based on Covariance Matrix Decomposition to Improve Multibaseline \& Multitemporal Interferometric SAR Processing," IEEE Transactions on Geoscience and Remote Sensing, vol. 53, no. 4, pp. 2050-2065, Apr. 2015.

[9] Ning Cao, Hyongki Lee, and Hahn Chul Jung, "A Phase-Decomposition-Based PSInSAR Processing Method," IEEE Transactions on Geoscience and Remote Sensing, vol. 54, no. 2, pp. 1074-1090, Feb. 2016.

[10] Richard Bamler and Philipp Hartl, "Synthetic aperture radar interferometry," Inverse Problems, vol. 14, no. 4, pp. R1-R54, Aug. 1998.

[11] Homa Ansari, Efficient High-Precision Time Series Analysis for Synthetic Aperture Radar Interferometry, Ph.D. thesis, German Aerospace Center and Technical Univercity of Munich, 2018. 